\title{
1,4-DI-N-OXIDE QUINOXALINE-2-CARBOXAMIDE: CYCLIC VOLTAMMETRY AND RELATIONSHIP BETWEEN ELECTROCHEMICAL BEHAVIOR, STRUCTURE AND ANTI- TUBERCULOSIS ACTIVITY
}

\author{
Elsa Moreno', Silvia Pérez-Silanes, Shravani Gouravaram², Abinav Macharam², Saioa \\ Ancizu $^{1}$, Enrique Torres ${ }^{1}$, Ignacio Aldana ${ }^{1}$, Antonio Monge ${ }^{1}$ and Philip W. Crawford ${ }^{2}$. \\ ${ }^{1}$ Neglected Diseases Section. Drug R\&D Unit., Centro de Investigación en Farmacobiología \\ Aplicada (CIFA), University of Navarra, C/ Irunlarrea s/n, 31008 Pamplona, Spain. \\ ${ }^{2}$ Department of Chemistry, Southeast Missouri State University, Cape Girardeau, Missouri \\ 63701, USA.
}

\section{INTRODUCTION}

Tuberculosis (TB) is an infectious bacterial disease mainly caused by Mycobacterium tuberculosis (M.tb). TB is a respiratory transmitted disease affecting nearly $32 \%$ of the world's population. The last data published by WHO established that there were an estimated 9.4 million incident cases and 11.1 million prevalent cases of TB in 2008, killing 1.3 million people worldwide [1]. The continuing emergence of multidrug-resistant and extremely drug-resistant strains of M.tb and the increasing incidences of the disease in immune-compromised patients highlights the urgent need for new drugs which could shorten the treatment duration and extend the range of effective TB treatment options [2, 3].

Quinoxaline derivatives show very interesting biological properties and several studies have been published demonstrating their capability to act not only as antibacterial and antimicrobial but also as antitumoral agents [4-7] (Fig. 1).

As a result of the anti-tuberculosis research project, our group has published several papers in which the synthesis and biological evaluation of a large number of quinoxaline and quinoxaline 1,4-di- $N$-oxide derivatives have been described [8-14]. These studies have facilitated a wide structure-activity relationship (SAR) analysis which lead us to design a group of seventy-seven 3-methylquinoxaline-2-carboxamide 1,4-di- $\mathrm{N}$-oxide derivatives that were prepared and tested against M.tb $[15,16]$. 
This project allowed us to observe that the lack of one or both of the $\mathrm{N}$-oxide groups generally led to the loss of the antimycobacterial activity $[17,18]$. The well-known $\mathrm{N}$-oxide bio-reduction process and its biological consequences seem to be essential for the biological activity displayed by heterocyclic di- $N$-oxides although little is known about their mode of action [19, 20].

Several studies have investigated and reported the electrochemical behavior of quinoxaline di$\mathrm{N}$-oxides and demonstrated reasonable correlations between electrochemical behavior, structure and drug activity [21-23]. Not only heterocyclic di- $N$-oxides but also antibacterial quinones and heterocyclic nitro derivatives have revealed that compounds showing less negative reduction potential values exhibited more powerful antibacterial activity [24].

These studies were performed under the hypothesis of the iminium theory. It was proposed that iminium species, which are believed to be generated metabolically in vivo, could take part in charge transfer (CT) processes, resulting in the formation of superoxide and generating toxic oxy radicals. At this point we decided to study the electrochemical properties of quinoxaline di$\mathrm{N}$-oxide derivatives which possess the suitable structural requirement and should be easily reduced due to their electrophilic nature and the stability of the resulting radical which is stabilized by resonance [22, 24, 25].

The experimental goal of this study was to determine the reduction potentials for several series of quinoxaline di- $\mathrm{N}$-oxides in order to obtain evidence of the relationship between the electrochemical behaviour and the anti-tuberculosis activity.

\section{EXPERIMENTAL}

\section{Chemical synthesis and anti-tuberculosis activity}

The methods for the synthesis of quinoxaline-2-carboxamide 1,4-di- $\mathrm{N}$-oxide derivatives were reported elsewhere $[15,16]$.

\section{Pharmacology}

In vitro evaluation of the anti-tuberculosis activity was carried out within the Tuberculosis Antimicrobial Acquisition \& Coordinating Facility (TAACF) screening program for the discovery of novel drugs for the treatment of tuberculosis. The Southern Research Institute coordinates the overall program under the direction of the U.S. National Institute of Allergy and Infectious Disease (NIAID). [26] 
The purpose of the screening program is to provide a resource whereby new experimental compounds can be tested for their ability to inhibit the growth of virulent Mycobacterium tuberculosis (M.Tbc.). Biological tests have been performed according to previously described methods.

The initial screening is conducted against M. Tbc. $\mathrm{H}_{37} \mathrm{Rv}$ (ATCC 27294) in BACTEC 12B medium using the Microplate Alamar Blue Assay (MABA).[32] Compounds are tested in ten 2fold dilutions, typically from $100 \mu \mathrm{g} / \mathrm{mL}$ to $0.19 \mu \mathrm{g} / \mathrm{mL}$. The $\mathrm{IC}_{90}$ is defined as the concentration effecting a reduction in fluorescence of $90 \%$ relative to controls. This value is determined from the dose-response curve using a curve-fitting program. Any $I_{90}$ value of $\leq 10 \mu \mathrm{g} / \mathrm{mL}$ is considered "Active" for antitubercular activity.

The VERO cell cytotoxicity assay is carried out in parallel with the TB Dose Response assay. After 72 hours exposure, viability is assessed using Promega's Cell Titer Glo Luminescent Cell Viability Assay, a homogeneous method for determining the number of viable cells in culture based on quantitation of the ATP present. Cytotoxicity is determined from the dose-response curve as the $\mathrm{CC}_{50}$ using a curve fitting program. Then the $\mathrm{CC}_{50}$ is divided by the $\mathrm{IC}_{90}$ for calculating a Selectivity Index $(\mathrm{SI})$ value. SI values of $\geq 10$ are considered for further testing.

\section{Electrochemistry}

Dimethylformamide (DMF) and tetrabutylammonium perchlorate (TBAP) were obtained commercially in the highest purity available and used without further purification. Ferrocene was obtained from the Aldrich Chemical Company. Cyclic voltammetric experiments were carried out on a $\mathrm{CHI}$ Instruments 630 voltammetric analyzer. Test solutions contained $0.5 \mathrm{mM}$ of the desired compound and $0.1 \mathrm{M}$ TBAP. Scan rates ranged from $50 \mathrm{mV} / \mathrm{s}$ to $500 \mathrm{mV} / \mathrm{s}$. Half-wave potentials $\left(E_{1 / 2}\right)$ were measured as the average of the cathodic and anodic peak potentials [27]. For first derivative cyclic voltammograms, $E_{p c}$ was determined at the point where the derivative curve crosses the baseline [27].

A three electrode cell was used for all electrochemical experiments, consisting of a Pt-disk (1.6 $\mathrm{mm}$ ) electrode, a Pt-wire auxilliary electrode, and a $\mathrm{Ag} / \mathrm{AgNO}_{3}(0.1 \mathrm{M}$ in acetonitrile) reference electrode (SRE). Deoxygenation of all solutions was accomplished by passing a gentle, constant stream of prepurified dinitrogen through the solution for 15 minutes and maintaining a blanket of the inert gas over the solution during the experiment. To account for daily variations in the reference electrode and liquid junction potentials, ferrocene (Fc) was added to each solution following measurements of the test compound, and used as an internal reference 
redox system [28]. All potentials are reported versus the ferrocene/ferrocinium $\left(\mathrm{Fc} / \mathrm{Fc}^{+}\right)$redox couple, i.e. $E_{p c}$, SRE $-E_{1 / 2, F c / F c}+$ or $E_{1 / 2}, S R E-E_{1 / 2, F c / F c}+$.

\section{RESULTS AND DISCUSSION}

\section{Electrochemical behavior}

The present study included several series of 37 quinoxaline-di- $N$-oxides. The redox properties of these substances were studied using cyclic voltammetry and first derivative cyclic voltammetry in DMF with TBAP as supporting electrolyte. Results are included as supplementary information and representative voltammograms are provided in Figure 1. All reductions were found to be diffusion controlled, as indicated by constant current functions at varying scan rates. $[27,29]$

For all compounds studied, two cyclic voltammetric waves were observed between -0.4 and $-1.9 \mathrm{~V}$ during reduction. The first voltammetric wave was irreversible under these conditions, with peak potentials, $\mathrm{E}_{\mathrm{pc}, 1}$, ranging from -1.21 to $-1.65 \mathrm{~V}$ (vs. $\left.\mathrm{Fc} / \mathrm{Fc}^{+}\right)$. For compounds 18-21, 23, 24, 27, 28, 31, and 33, this wave appeared as a shoulder to the second wave in the voltammogram. With a few exceptions (see following), the second voltammetric wave was quasireversible under the conditions used in the study, with peak potentials, $E_{p c, 2}$, ranging between -1.39 and $-1.92 \mathrm{~V}$, and half-wave potentials ranging between -1.42 and $-1.80 \mathrm{~V}$. For compounds $1,8,16,17$, and 31 this voltammetric wave was not very well defined, appearing as a shoulder in the cyclic voltammogram. Values of $\Delta \mathrm{E}_{\mathrm{p}}$ for this wave $(100 \mathrm{mV} / \mathrm{s})$ were generally greater than theoretical for a reversible, one-electron reduction, and increased with increasing scan rate. For compounds $3, \mathbf{9}, \mathbf{1 2}, \mathbf{2 2}$, and $\mathbf{2 6}$, the second voltammetric wave was irreversible under the conditions utilized in this study. Calculated $i_{p a} / i_{p c}$ ratios for compounds 2, 5-8, 11, 13, 15, $21,23,24,27,29-35$, and 37 were close to unity (0.61 to 1.24$)$ at all scan rates, indicating the formation of relatively stable reduction products for this process on the time scale of the voltammetric scans. Current ratios that deviated significantly from unity $(0.24$ to 0.55$)$ were observed for all other derivatives, indicative of greater kinetic or other complications following the reduction [29]. No reduction waves were observed at potentials negative of the second voltammetric wave for these quinoxaline-1,4-dioxide derivatives under the conditions used in this study, with the exception of compounds 3-5, 9-12, 22, 26, 33 and 37. For the latter compounds, a third irreversible reduction wave was observed at potentials ranging from -1.885 to $-2.414 \mathrm{~V}$. 
Examination of the data indicates the influence of quinoxaline structure on potential. The 37 compounds can be broken down into 9 different analogues based on structure, with the mono chloro and methyl substituted quinoxalines existing as mixtures of 6- and 7-substituted positional isomers. Within each analogous series, replacement of the 6-/7-hydrogen atom with a single electron-withdrawing substituent group, i.e. chloro, fluoro, and trifluoromethyl, resulted in a positive shift in the peak potentials for both voltammetric waves, whereas replacement of the 6-/7hydrogen atom with a single electron-releasing methyl group resulted in a negative shift. As an example, comparison of the potentials for compounds 13 and 15 shows that replacement of the hydrogen atom with the chloro group resulted in a $+109 \mathrm{mV}$ potential shift for the first voltammetric wave, and a $+76 \mathrm{mV}$ shift in the second voltammetirc wave $\left(+105 \mathrm{mV}\right.$ shift in $\left.\mathrm{E}_{1 / 2}\right)$. Replacing the hydrogen atom in compound $\mathbf{1 3}$ with the methyl group in compound $\mathbf{1 4}$ resulted in a $-20 \mathrm{mV}$ potential shift for the first voltammetric wave, and a $-10 \mathrm{mV}$ shift in the second voltammetric wave (-8 $\mathrm{mV}$ shift in $\left.E_{1 / 2}\right)$. Similarly, comparison of compound $\mathbf{6}$ with compounds $\mathbf{1 0}$ and $\mathbf{1 1}$ shows that substituting the 7-hydrogen with trifluoromethyl or fluoro groups results in a $+186 \mathrm{mV}$ and $+74 \mathrm{mV}$ shift in the first voltammetric wave, respectively. The reduction potentials for the quinoxaline derivatives within each analogue generally fit the modified Hammett equation, $\Delta \mathrm{E}_{1 / 2}=\rho_{\pi \mathrm{R}} \sigma_{\mathrm{x}}$ (correlation coefficients ranged from 0.89 to 1 ), where $\sigma_{x}$ is the polar inductive electronic substituent constant taken as the average of the sum of $\sigma_{\mathrm{m}-\mathrm{x}}$ and $\sigma_{\mathrm{p}-\mathrm{x}}$, and $\rho$ is the reaction constant [30]. (Figure 2) (For quinoxaline derivatives substituted in the benzene ring, the use of $\left(\sigma_{\mathrm{m}-\mathrm{x}}+\sigma_{\mathrm{p}-\mathrm{x}}\right) / 2$ has been recommended[31].) These effects are consistent with facilitation of reduction by a positive charge at the site of reduction [30], in keeping with previous reports [22, $25,30,32,34]$. Addition of a second electron-withdrawing group, i.e. chloro or fluoro, enhances this effect. For example. comparison of the 6-,7-dichloro derivatives $(3,9,22,26,33$ and 37$)$ with the corresponding 6-/7-chloro derivatives $(2,8,21,25,32$ and 36) shows that addition of the second chloro group shifts the potential of the first voltammetric wave positively by +81 to +271 $\mathrm{mV}$, and that of the second voltammetric wave by +121 to $+395 \mathrm{mV}$. No apparent correlation was observed between substitution in the amide side chain and potential (cf. 1, 14, 17, 20, 24, 28, 31 and 35). In the latter case, the substituents are presumably too far removed from the site of reduction.

\section{Relationship between electrochemical behavior and anti-tuberculosis activity}

With regards to the mechanism, heterocyclic di- $N$-oxides possessing the diiminium structure are expected to undergo one electron reduction to form a radical anion [32, 35-38] 
(Figure 3), and in fact reduction of quinoxaline 1,4-di- $\mathrm{N}$-oxide in DMF has been shown via ESR and cyclic voltammetry to involve the nitrone function to form the radical anion [33]. The electrochemical reductions of quinoxaline $1,4-\mathrm{di}-\mathrm{N}$-oxides in aprotic solvent systems have been reported previously [22, 25, 32-34]. In most cases, a reversible reduction wave attributed to reduction of the $\mathrm{N}$-oxide functionality has been reported in DMF in the vicinity of those observed in the present study [22, 25, 32, 33]. For the compounds in the present study, electrochemical reduction in DMF is consistent with reduction of the $\mathrm{N}$-oxide functionality to form a radical anion as well. The second reduction wave observed could be due to the formation of the dianion, or to reduction of the product formed from a chemical step involving the radical anion [34]. The latter is supported by the irreversibility of the first reduction wave. Kaye and Stonehill pointed out that an objection to the iminium theory is that some potentials observed are too negative although several reports indicate that reduction potential in vivo may be better than in vitro [24], and $\mathrm{N}$ oxides are known to undergo bioreduction $[19,20]$.

No absolute correlation can be established between the reduction potential and the biological activity. Nevertheless, within the compounds which have shown biological activity (112) (Table 1) it can be said that the less negative the reduction potential is, the more active the compounds are. In fact, for compounds showing a $E_{p c, 1<-1.6 V}$ the $I_{90}$ increases almost

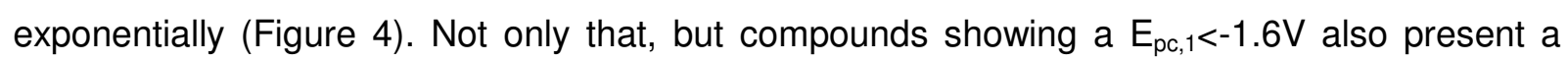
selectivity index $(\mathrm{SI})$ much lower than compounds with a reduction potential that is less negative (Figure 5). Of course, absolute correlation between electrochemical behavior and antituberculosis activity is not reasonable as many other factors should be considered: solubility, metabolism, absorption or site binding among others. The results support the participation of charge transfer processes in the mechanism of action of quinoxaline di- $N$-oxides; however, the exact mechanism was not investigated further.

\section{CONCLUSIONS}

Thirty-seven quinoxaline di- $N$-oxides have been studied and all of them showed two cyclic voltammetric waves between -0.4 and $-1.9 \mathrm{~V}$ during reduction. The first voltammetric wave was irreversible for all the studied compounds under the conditions of the study. A relationship between electrochemical behavior and quinoxaline structure can be established and it can be said that the insertion of an electron-withdrawing group on the quinoxaline ring results in a less negative reduction potential and makes the bio-reduction more facile. Nevertheless, no 
relationship could be found between substitution on the amide chain and the reduction potential. These results are expected to offer new insights into the study of the mechanism of action for future antitubercular drugs.

\section{ACKNOWLEDGMENTS}

This work has been carried out with the financial support of the PIUNA project from University of Navarra. We also wish to express our gratitude to the Tuberculosis Antimicrobial Acquisition \& Coordinating Facility (TAACF) for the evaluation of the anti-tuberculosis activity through research and development contracts. E. M. is indebted to the La Rioja Government for a grant. 


\section{REFERENCES}

1. WHO. Fact sheet №104 March 2010.

2. Global Tuberculosis Control WHO REPORT 2009, http://www.who.int/tb/publications/global_report/2009/pdf/full_report.pdf

3. Multidrug and extensively drug-resistant TB. 2010 Global report on surveillance and response. WHO/HTM/TB/2010.3

4. K.L. Leverkusen, U. Eholzer, R. Nast, F. Seng, US3660398 (1972).

5. M. Abu El-Haj, DE2316765 (1973).

6. J.G. Frienlink, NL6504563 (1966).

7. K.M. Amin, M.F. Ismail, E. Noaman, D.H. Soliman, Y.A. Ammar, Bioorg. Med. Chem. 14 (2006) 6917e6923.

8. Ancizu S, Moreno E, Torres E, Burguete A, Pérez-Silanes S, Benítez D, Villar R., Solano B., Marín A., Aldana I., Cerecetto H., González M., Monge A. Molecules 2009;14, 2256.

9. Jaso A, Zarranz B, Aldana I, Monge A. J. Med. Chem. 2005, 48, 2019.

10. Jaso A, Zarranz B, Aldana I, Monge A. Eur.J.Med.Chem. 2003, 38, 791.

11. Vicente E, Pérez-Silanes S, Lima LM, Ancizu S, Burguete A, Solano B, Villar R., Aldana I., Monge A. Bioorg.Med.Chem. 2009, 17, 385.

12. Vicente E, Villar R, Burguete A, Solano B, Pérez-Silanes S, Aldana I, et al. Antimicrob. Agents Chemother. 2008, 52, 3321.

13. Villar R, Vicente E, Solano B, Pérez-Silanes S, Aldana I, Maddry JA, Lenaerts AJ., Franzblau SG., Cho SH., Monge A., Goldman RC. J. Antimicrob. Chemother. 2008, 62, 547.

14. Zarranz B, Jaso A, Aldana I, Monge A. Bioorg.Med.Chem. 2003, 11, 2149.

15. Moreno, E.; Ancizu, S., Pérez-Silanes S., Torres E., Aldana I., Monge, A. Eur. J. Med. Chem. 2010, 45, 4418.

16. Ancizu, S.; Moreno, E., Solano B., Villar R., Burguete A., Pastor E., Pérez-Silanes S., Aldana I., Monge A. Bioorg. Med. Chem. 2010, 18, 2713

17. Ortega MA, Montoya ME, Jaso A, Zarranz B, Tirapu I, Aldana I, Monge A. Pharmazie 2001, 56, 205.

18. Ortega MA, Sainz Y, Monge-Vega A, Jaso A, Zarranz B, Aldana I, Monge A. Arznelm.Forsch./Drug. Res. 2002, 52, 113.

19. Elwell, J.H.; Aiim, B.G.; Evans, J.W.; Brown, J.M. Biochem. Pharmacol. 1997, 54,249.

20. Cerecetto, H.; González, M. Mini-Rev. Med. Chem. 2001, 1, 219. 
21. Ryan M.D.; Scamehorn R.G., Kovacic, P. J. Pharmaceut. Sci. 1985, 74, 492.

22. P. W. Crawford, R. G. Scamehorn, U. Hollstein, M. D. Ryan, and P. Kovacic, Chem.-Biol. Interactions 1986, 60, 67.

23. R.P. Mason. Free-radical intermediates in the metabolism of toxic chemicals. In: Free Radicals in Biology, Volume 5 (W.A. Pryor, ed.), pp 161-222, Academic Press, New York 1982.

24. Ames, J.R.; Ryan, M.D.; Kovacic, P. Journal of Free Radicals in Biology \& Medicine, vol 2, 1986, 377-391.

25. J. R. Ames, M. A. Houghtaling, D. L. Terrian, Electrochim. Acta 37, 1433 (1992).

26. www.taacf.org

27. P. H. Rieger, Electrochemistry, 2nd edition, Chapman and Hall, New York (1994).

28. G. Gritzner and J. Kuta, Pure Applied Chem. 56, 461 (1984).

29. A. J. Bard and L. R. Faulkner, Electrochemical Methods: Fundamentals and Applications, Wiley, New York (2001).

30. P. Zuman, Substituent Effects in Organic Polarography, Plenum Press, New York (1967).

31. M. P. Strier and J. C. Cavagnol, J. Am. Chem. Soc. 80, 1565 (1958).

32. M. D. Ryan, R. G. Scamehorn, and P. Kovacic, J. Pharmaceut. Sci. 74, 492 (1985).

33. H. Miyazaki, Y. Matsuhisa, and T. Kubota, Bull. Chem. Soc. Jpn. 54, 3850 (1981).

34. K. R. Barqawi and M. A. Atfah, Electrochim. Acta 32, 597 (1987).

35. P. Kovacic, Kem. Ind. 33, 473 (1984).

36. Priyadarsini KI., Dennis MF., Naylor MA., Stratford MRL., Wardman P. J. Am. Chem. Soc. 1996, 118, 5648.

37. Gomes JRB., Ribeiro da Silva MDMC., Ribeiro da Silva MAV. Chem. Physics Letters. 2006, $429,18$.

38. Junnotula V., Rajapakse A., Arbillaga L., López de Cerain A., Solano B., Villar R., Monge A., Gates KS. Bioorg. Med. Chem. 2010, 18, 3125. 